one part of proximal convoluted and straight tubules has been indicated ab. sence of glutaminase activity. In normal rat kidney, moderate glutaminase activity activated by bicarbonate in the proximal convoluted and straight tubules was present. There was the absence of the activity in the proximal convoluted tubules of injured kidney. The collecting ducts and ascending and descending thin limbes showed no reaction. Distal convoluted tubules in acidotic rats showed the high activities of the both glutaminases. Moreover, the data of other enzyme activities are summarized in Table 1.

Of particular interest were the high activities of both glutaminases and glutamic dehydrogenase observed in rats in the straight convoluted and distal tubules and the strong activities of glutamic oxalacetic transaminase and carbonic anhydrase in the proximal convoluted tubules.

\title{
References
}

1) Richterich, R.W. and Goldstein, L., Am. J. Physiol., $195: 316,1958 . \quad$ 2) Karnovsky, M. J. and Himmelhoch, S.R., Am. J. Physiol., $201: 768,1961 . \quad 3)$ Katunuma, N., 17 th Symp. of Enzyme Chemistry, 407, 1965, Tokushima. 4) Lotspeich, W.D. and Pitts, R.F., J. Biol. Chem., $168: 611,1947 . \quad 5)$ Kishino, Y., Medicine and Biology, $70: 267,1965 . \quad 6)$ Nachlas, M.M. et al, J. Biophy. and Biochem. Cytol., $4: 29,1958$. 7) Häusler, G., Histochemie, 1: 29, 1958.

\section{Leuco-Malachite Green Staining for Ceroid}

\author{
Ryuei Maeda, Reiko Takada, Tatsuji Hagihara and Ichiro Yamagata \\ Department of Pathology, Kansai Medical School, Moriguchi
}

For differentiating histochemically ceroid form lipofuscins, acid fast fuchsin stain, Schmorl reaction or chrome alum hematoxylin stain have been most of ten employed in paraffin sections, but these methods have been found unsatisfactory. A $0.05 \%$ nile blue stain $(\mathrm{pH} 3.0)$ is more useful to differentiate both pigments; nevertheless, this method may be not said to be quitely satisfactory. The present staining method is an outcome of further investigation of twentyfour leuco-dyes containing the triphenylmethane (15), oxazine (2), thiazine (3), azine (2), xanthine (1), and quinone imine (1) grous.

The procedure is as follows:

1. Formalin fixation is preferable.

2. Cut the paraffin block section.

3. Immerse for $18-24$ hours at the room temperature in the following reagent.

Dissolve $150 \mathrm{mg}$. leuco-malachite green (Grübler Co.) in $14 \mathrm{cc}$. heated absolute ethyl alcoho 1 , add $20 \mathrm{cc}$. distilled water, $6 \mathrm{cc}$. glacial acetic acid and $8 \mathrm{cc}$. of $0.8 \% \mathrm{KIO}_{4}$ in $0.3 \% \mathrm{HNO}_{3}$. 


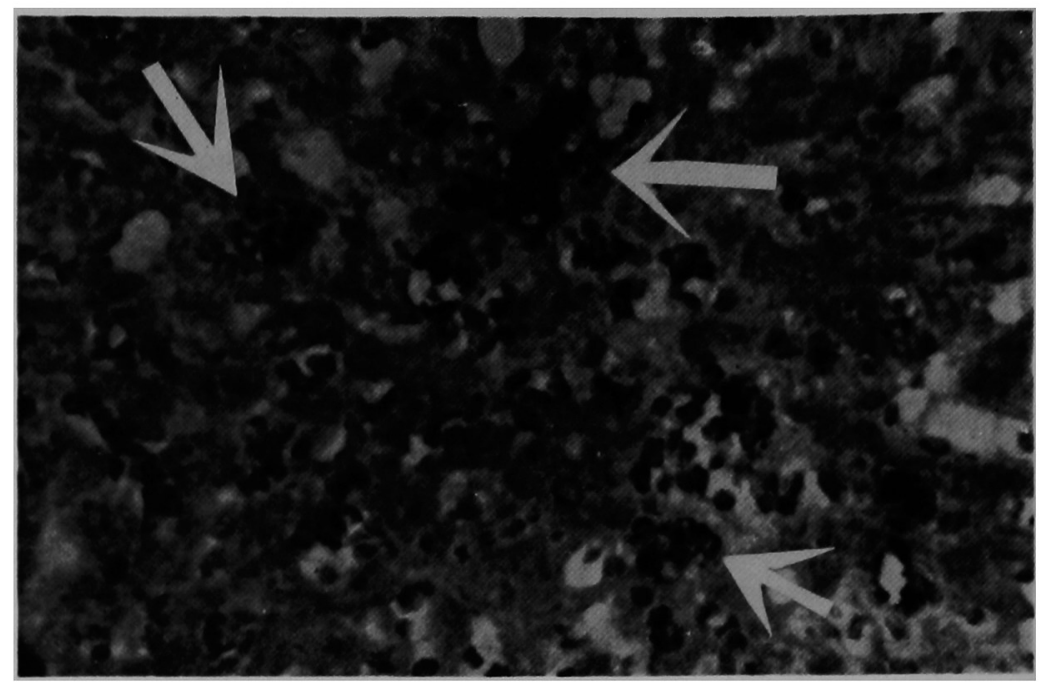

4. Wash in water repeatedly.

5. Counterstain in $1 \%$ aqueous eosin for several seconds.

6. Rinse briefly in water.

7. Remove excess water gently with the use of filter paper.

8. Dehydrate in alcohol quickly taking less than two minutes, then clear in xylene, and mount in balsam.

Results : Ceroid presents an emerald green color. Mast cell granules are also stained; lipofuscins and hemosiderin unstained in the natural brown.

Note : In preparing the staining solution (3), first, malachite green should be dissolved in heated alcohol, secondly, its solution presents cloudiness when distilled water is added but with an addition of glacial acetic acid it turns clear. The solution of $\mathrm{KIO}_{4}$ is routinely added. This solution should be freshly made each time.

\section{References}

1) Endicott, K.M., and Lillie, R.D., Am. J. Path., 20, 194, $1944 . \quad$ 2) Lille, R.D., Stain Technol., 31, 151, $1956 . \quad$ 3) Takada, R., Tr. Soc. Path. Jap., 49, 1035, 1960. 4) Takada, R., Hagihara, T., Kanazawa, K., and Kondo, T., Tr. Soc. Path. Jap., 54, 1965. In press.

\section{Explanation of a figure}

Photomicrograph of liver section of an adult Wistar rat fed by a vitamin E-defficient diet for 294 days. Leuco-malachite green staining. Darkest granules shown by arrows indicating accumulation of ceroid. $\times 400$. 